\title{
Varied Clinical Presentation and Management of Paediatric Vallecular Cyst
}

Jyoti Singh, ${ }^{1}$ "Neha Jain, ${ }^{1}$ Mamta Jajoo, ${ }^{2}$ Suparna Roy, ${ }^{1}$ Ekta Narang, ${ }^{1}$ Nidhi Mahajan ${ }^{3}$

\begin{abstract}
The current article aims to highlight the varied presentation and management of vallecular cysts. We report three children, aged four years, 11 months and three days, diagnosed with vallecular cyst presenting to the Chacha Nehru Bal Chikitsalaya hospital, New Delhi, India in 2018 and 2019. They were reviewed retrospectively for clinical presentation, diagnostic tools and treatment options. All three cases had respiratory and feeding difficulties. The diagnosis in all of the three cases was made with laryngoscopy (flexible/direct) and imaging. All three patients were treated with the transoral approach aimed at the excision of the cyst using cold instruments. The postoperative period was uneventful. No recurrences were observed during the long-term follow-up. Vallecular cysts should be considered as one of the differentials in children with respiratory distress and dysphagia despite being a rare anomaly. A direct transoral approach is recommended for the excision of the vallecular cyst as it is a safe and reliable method with no recurrences to date.
\end{abstract}

Keywords: Benign Cyst; Dyspnea; Dysphagia; Laryngoscopy; Case Report; India.

A VALLECULAR CYST IS A UNILOCULAR CYSTIC mass which contains clear and non-infected fluid. ${ }^{1}$ Vallecular cysts are rare anomalies presented with respiratory distress, dysphagia and failure to thrive and they can lead to potentially lifethreatening conditions and sudden airway obstruction. ${ }^{2}$ Symptoms such as chest retraction, apnoeic/cyanotic episodes, choking, postprandial vomiting, coughing and hoarse cry are also seen occasionally. ${ }^{1,3}$ Any infant showing such symptoms should be considered to be having laryngomalacia, upper airway anatomical anomalies or upper airway obstructive lesions such as haemangioma, cystic hygroma, teratoma, hamartoma, dermoid cyst, thyroglossal duct cyst or thyroid remnant. ${ }^{1}$

Nasopharyngolaryngoscopy and imaging are required to confirm the diagnosis and to observe the extension of the cyst. ${ }^{1}$ The treatment options include cyst aspiration, marsupialisation deroofing of the cyst wall and complete excision. ${ }^{4}$

This case series details the presentation and management of vallecular cysts in three paediatric cases. Informed consent was taken from all the three patients for publication of the article.

\section{Case One}

A four-year-old male child presented to the Ear Nose Throat (ENT) Clinic at the Chacha Nehru Bal Chikitsalaya hospital, New Delhi, India in 2018, with progressive deglutition problems, intermittent odynophagia and persistent dry cough for a year before he presented to the clinic. His oral examination was normal. Flexible naso-pharyngo-laryngoscopy showed a pale pink-coloured swelling over the lingual surface of the epiglottis [Figure 1A]. The contrast-enhanced computed tomography (CECT) scan showed an ovoid non-enhancing cystic lesion at the level of the epiglottis [Figure 2]. An endoscopy-assisted cyst excision was performed via the trans-oral route under general anaesthesia using cold instruments and electrical cautery [Figure 1B]. The histopathological examination revealed a cyst lined by pseudostratified columnar epithelium with dense lymphocytic infiltrate within the sub-epithelium [Figure 1C].

Analgesics, antibiotics and dexamethasone were administered in the postoperative period and the patient was discharged after 72 hours. No sign of local recurrence was seen during follow-up at two years.

\section{Case Two}

An 11-month-old male infant presented to the Chacha Nehru Bal Chikitsalaya hostpital, New Delhi, India in 2019 with respiratory distress, choking spells and frequent regurgitation of feeds. The child had noisy breathing since one month of age and keeping laryngomalacia as a possible diagnosis in mind, the primary hospital had planned for conservative management. The patient did well till eight months of age. During an examination, the patient got agitated, had an apnoeic spell requiring resuscitation and was subsequently intubated. An urgent computed tomography (CT) scan was performed to evaluate 

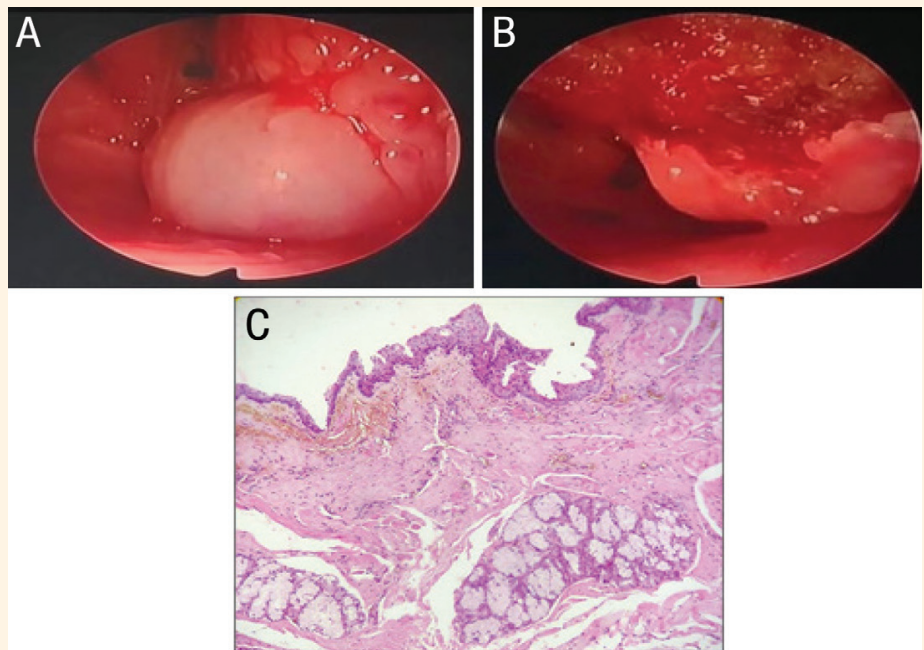

Figure 1: A: Flexible nasopharyngolaryngoscopy scans of a four-year-old child showing a pale pink-coloured swelling over the lingual surface of the epiglottis. B: Postoperative endoscopy scan showing excision of the cyst with a cold instrument and the base cauterised. C: Microscopic section at x40 magnification showing a cyst lined by stratified squamous epithelium and the cyst wall showing mucous glands with no detection of nuclear atypia.

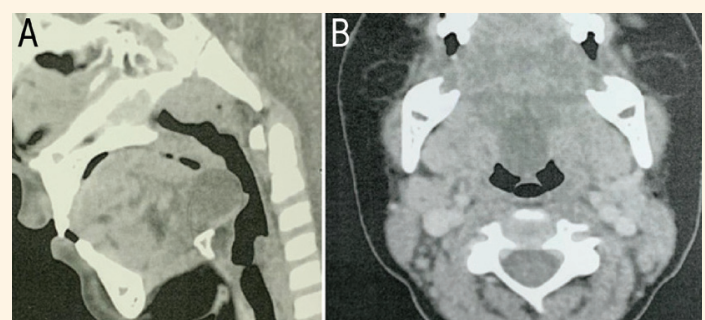

Figure 2: Contrast-enhanced computed tomography (CECT) scans of a four-year-old child showing an ovoid cystic mass at the base of the tongue and epiglottis. A: A lateral CECT slice showing minimal narrowing of the airway. B: An axial CECT slice showing the cyst on the lingual surface of the epiglottis.
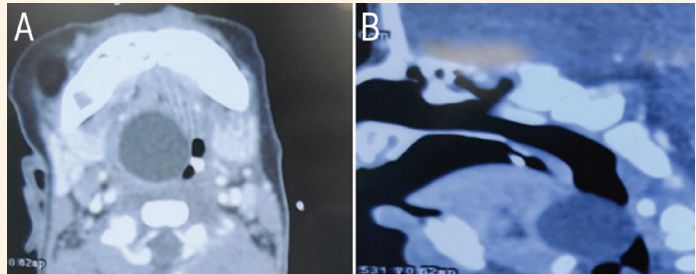

Figure 3: Computed tomography scans of an 11-monthold male infant showing (A) an axial view of a cystic ovoid mass measuring $17 \times 8.9 \mathrm{~mm}$ embedded in the base of the tongue and (B) a lateral view revealing compromised airway due to tongue base cystic lesion.

the upper airway and a cystic mass, $17 \times 8.9 \mathrm{~mm}$ in diameter, was seen embedded in the base of the tongue [Figure 3].

The patient was shifted to the operation theatre (OT). General anaesthesia was administered and when direct laryngoscopy was performed, a large cystic swelling was found at the base of the tongue. The molar retractor was positioned and retraction

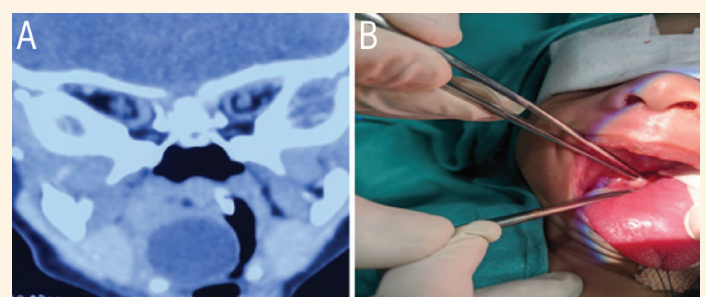

Figure 4: Images of a full-term, three-day-old male neonate including (A) a computed tomography scan showing the ovoid non-enhancing cyst attached to the right lateral wall of the vallecula, causing airway narrowing and (B) a clinical image showing cyst removal via the trans-oral approach wherein the tongue is retracted and the vallecular cyst is clasped with forceps.

sutures were placed to retract the tongue. Deroofing of the cyst wall was done and the remnant of the cyst wall was cauterised. The patient was extubated and observed overnight in the intensive care unit (ICU). The histopathological examination confirmed the diagnosis of a benign cystic lesion. The child recovered well with no recurrence during the two-year followup.

\section{Case Three}

A full-term, three-day-old male neonate was referred to the ENT clinic from the neonatal ICU at the Chacha Nehru Bal Chikitsalaya hospital, New Delhi, India in 2019 with a history of inspiratory stridor, choking and difficulty in feeding since birth. On examination, the neonate was found to be in respiratory distress. The patient was kept on continuous positive airway pressure and saturation was maintained above $95 \%$. The oral examination was normal. A gentle laryngoscopic 
examination showed a huge vallecular cyst obscuring the endo-larynx. The urgent CT scan that was performed confirmed the origin and extension of the cyst [Figure 4A]. Antibiotics, dexamethasone and histamine-2 blockers were given in the preoperative period.

The patient was taken to the OT, but the endolarynx was difficult to visualise with awake fibreoptic intubation due to the presence of a large cyst and anteriorly placed larynx. Two $\mathrm{mL}$ of fluid was then aspirated from the cyst which aided in successful intubation. Surgery was planned to be performed via the transoral route. The tongue was retracted with sutures (Silk 2-0) and the Hopkins 30-degree endoscope was used. The cyst was clasped with forceps and excised [Figure 4B].

Complete excision of the cyst could not be ascertained due to the attachment of the cyst to the lateral wall of the vallecula and inadequate exposure. The base of the cyst was cauterised and the wound was left to heal by secondary intention. The neonate was shifted back to the ICU and observed overnight. The very next day, the neonate was extubated and significant improvement was observed as far as the patient's respiratory distress was concerned. The diagnosis of a cyst with columnar epithelial lining was confirmed on biopsy. There was no recurrence during 10 month follow up after the surgical procedure.

Informed written consent was obtained for all surgical procedures and for publication of case series without disclosing patient identity . Records are kept in the Medical Record Department of our institute.

\section{Discussion}

This article described three cases of paediatric vallecular cysts with varied presentations: a newborn infant presenting with inspiratory stridor and failure to thrive, a child on conservative treatment for laryngomalacia and a toddler presenting with chief complaints of dysphagia.

Abercrombie described vallecular cysts in $1881 .{ }^{5}$ Vallecular cysts have been given various names such as congenital and ductal cyst, mucus retention cyst, epiglottic cyst and base of tongue cyst. ${ }^{3,6}$ The incidence of laryngeal cysts is approximately $5 \%$ of all benign laryngeal lesions and vallecular cysts make $10.5 \%$ of all congenital laryngeal cysts. ${ }^{7,8}$ Thus, a vallecular cyst is a rare entity. ${ }^{9}$

Laryngeal cysts have been classified into ductal, saccular and thyroid foraminal subtypes by De Santo. ${ }^{6}$ Ductal cysts are the most common and are frequently found in the vallecula and true vocal cords. Cysts can be further classified based on their histology and location as congenital cysts, retention cysts, inclusion cysts and lymphoepithelial cysts. ${ }^{8}$

Vallecular cysts can be found at any age, but their presentation differs depending on an individual's age and cyst size. They are usually asymptomatic when small in size; however, when they increase in size and/or become infected, they may cause respiratory, feeding and speech difficulties. ${ }^{10}$ Inspiratory stridor and dyspnoea are most commonly seen after birth, and failure to thrive with feeding difficulties is more commonly noted among older children. ${ }^{11}$ Symptoms such as chest retraction, apnoeic/cyanotic episodes, choking, postprandial vomiting, coughing and hoarse cry are also seen occasionally. ${ }^{1-3}$

Vallecular cysts may also cause supraglottic obstruction due to their mass effect and, more importantly, by displacement of the epiglottis posteriorly and inferiorly which may present as episodes of apnoea and cyanosis. ${ }^{12}$ Increased size of these cysts progressively interferes with swallowing, subsequently leading to poor weight gain and failure to thrive. ${ }^{13}$ Due to the anatomical location of the cyst and the small airway in the case of an infant, there is a higher risk of sudden airway obstruction. ${ }^{14}$ Around $12-45 \%$ of patients with laryngomalacia may have coexisting airway abnormalities such as a laryngeal cyst. ${ }^{15}$ Subsequent increase in inspiratory negative pressure leads to supraglottic prolapse and the development of laryngomalacia. ${ }^{2}$ Hence, vallecular cyst should be considered in the differential diagnosis of congenital stridor in infants along with laryngomalacia, vocal cord palsy and subglottic haemangioma. ${ }^{11-14,16}$

A combination of a detailed history and radiological and endoscopic assessment provides the necessary information that can confirm the diagnosis of vallecular cyst. ${ }^{3}$ Lateral radiography, flexible nasopharyngolaryngoscopy, direct laryngoscopy, $\mathrm{CT}$, magnetic resonance imaging (MRI) and ultrasonography (USG) of the neck are helpful tools in the assessment of vallecular cyst. ${ }^{3}$

Flexible endoscopy is an initial screening tool. Direct laryngoscopy is used in securing the airway, detection of other lesions and surgical management. ${ }^{3}$ CECT scan or MRI are essential to determine the size, site, anatomic dimensions and extension of the cyst so that the surgical resection can be planned. MRI is a diagnostic modality of choice, but it is time-consuming, requires sedation and is expensive because of which it is not widely used in children with compromised airways obstruction. ${ }^{17}$

Laryngoscopy (flexible/direct) was performed in each of the said three cases to confirm the diagnosis along with a CECT scan to determine the dimensions 
and location of each cyst before planning the respective surgeries. It is to be noted that prenatal diagnosis of this condition is possible with ultrasound scanning or foetal MRI which can help surgeons secure the infant's airway during birth. ${ }^{3}$

A limited number of case reports and series of vallecular cysts have been published stating different treatment modalities. Surgical options for dealing with vallecular cysts in infants and children include cyst aspiration, marsupialisation (i.e. deroofing) and extirpation (i.e. resection and excision). ${ }^{4}$ However, aspiration of cyst is not advocated for its high recurrence rate.9 Hence, aspiration should be considered either as a palliative procedure or as an initial step in cases of difficult intubation for decompression of the cyst. ${ }^{9,12}$

A definitive treatment of vallecular cyst is marsupialisation or extirpation. ${ }^{4,9}$ In a retrospective analysis, $\mathrm{Li}$ et al. found successful outcomes from marsupialisation using micro-instruments, electrocautery, potassium-titanyl-phosphate (KTP) laser and micro-debrider and a $15 \%$ recurrence rate. ${ }^{3}$ Gutierrez et al. reported success and safety with marsupialisation using cup forceps and $\mathrm{CO}_{2}$ laser. ${ }^{1}$ Hsieh et al. used a $\mathrm{CO}_{2}$ laser in for treating vallecular cysts of 33 patients with successful outcomes and no recurrence. ${ }^{18}$ The use of a $\mathrm{CO}_{2}$ laser is recommended because these are less invasive and if these are used, the healing can be fast. ${ }^{18} \mathrm{~A}$ vallecular cyst may often be approached via the direct transoral route or by using suspension laryngoscopy. It is only in rare cases that an external approach is required because recurrent lesions and large cysts need to be extirpated. ${ }^{3}$

This article has described a simple, direct and transoral approach for managing vallecular cyst without any special equipment. This approach can be safely adopted for infants and the recurrence rate consequent to using this approach is minimal. Chen et al. found no recurrence in seven patients who underwent complete cyst excision via the transoral surgical approach. ${ }^{19}$ Similarly, in the above-described cases too, no recurrence has been observed so far during the follow-up period of the three cases which has ranged from 10 months to two years.

In the currently reported case series, oral fibreoptic intubation was done in case one and case two was intubated in an emergency. In case three, fibreoptic intubation was difficult, so the cyst was aspirated with a large-bore needle, following which the child was intubated orally. However, in general, it must be noted that tracheostomy may be needed in a few cases to secure the airway. ${ }^{9}$

\section{Conclusion}

Vallecular cysts are rare and the challenge in making an early diagnosis, keeping laryngomalacia in mind, has been ascertained in the above case series. It is important to state that each case requires an individualised approach. The direct transoral approach for excision of the vallecular cyst is recommended as a safe and reliable method based on our experience of with no recurrences to date. Further randomised studies are required to determine the best diagnostic and treatment options for this vallecular cysts.

\section{AUTHORS' CONTRIBUTIONS}

JS and NJ collected the data. NJ drafted the manuscript and handled the designing and formatting. EN formulated the abstract. JS and SR revised the manuscript while MJ and EN proofread the manuscript. All authors approved the final version of the manuscript.

\section{References}

1. Gutierrez JP, Berkowitz RG, Robertson CF. Vallecular cysts in newborns and young infants. Pediatr Pulmonol 1999; 27:282-5. https://doi.org/10.1002/(sici)1099-0496(199904)27:4<282::aidppul10>3.0.co;2-g.

2. Yao TC, Chiu CY, Wu KC, Wu LJ, Huang JL. Failure to thrive caused by the coexistence of vallecular cyst, laryngomalacia and gastroesophageal reflux in an infant. Int $\mathrm{J}$ Pediatr Otorhinolaryngol 2004; 68:1459-64. https://doi.org/10.1016/j. ijporl.2004.06.004.

3. Li Y, Irace AL, Dombrowski ND, Perez-Atayde AR, Robson CD, Rahbar R. Vallecular cyst in the pediatric population: Evaluation and management. Int J Pediatr Otorhinolaryngol 2018; 113:198-203. https://doi.org/10.1016/j.ijporl.2018.07.040.

4. Hsieh WS, Yang PH, Wong KS, Li HY, Wang EC, Yeh TF. Vallecular cyst: an uncommon cause of stridor in newborn infants. Eur J Pediatr 2000; 159:79-81. https://doi.org/10.1007/ pl00013809.

5. Abercrombie J. Congenital cyst in larynx. Trans Pathol Soc Lond $1881 ; 32: 33-4$.

6. De Santo LW, Devine KD, Weiland LH. Cysts of the larynxclassification. Laryngoscope 1970; 80:145-76. https://doi. org/10.1288/00005537-197001000-00013.

7. RamesarK,AbizzatiClaryngealcysts:clinical relevanceandmodified working classification. J LaryngOtol 1988; 102: 923-925.

8. Arens C, Glanz H, Kleinsasser O. Clinical and morphological aspects of laryngeal cysts. Eur Arch Otorhinolaryngol 1997; 254:430-6. https://doi.org/10.1007/bf02439974.

9. Mitchell DB, Irwin BC, Bailey CM, Evans JN. Cysts of the infant larynx. J Laryngol Otol 1987; 101:833-7. https://doi. org/10.1017/s0022215100102828.

10. Berger G, Averbuch E, Zilka K, Berger R, Ophir D, Saba K. Adult vallecular cyst: Thirteen-year experience. Otolaryngol Head Neck Surg 2008; 138:321. https://doi.org/10.1016/j. otohns.2007.12.008. 
11. Tuncer U, Aydogan LB, Soylu L. Vallecular cyst: a cause of failure to thrive in an infant. Int J Pediatr Otorhinolaryngol 2002; 65:133-5. https://doi.org/10.1016/s0165-5876(02)00078-2.

12. LaBagnara J. Cysts of the base of the tongue in infants: An unusual cause of neonatal airway obstruction. Otolaryngol HeadNeck Surg 1989;101:108-11.https://doi.org/10.1177/0194 59988910100119.

13. Gluckman PGC, Chu TWF, Van Hasselt CA. Neonatal vallecular cysts and failure to thrive. J Laryngol Otol 1992: 106:448-9. https://doi.org/10.1017/s0022215100119796.

14. Lewison MM, Lim DT. Apnea in the supine position as an alerting symptom of a tumor at the base of the tongue in small infants. J Pediatr 1965; 60:1092-3. https://doi.org/10.1016/ s0022-3476(65)80096-8.

15. Mancuso RF, Choi SS, Zalzal GH, Grundfast KM, Laryngomalacia. The search for the second lesion, Arch. Otolaryngol. Head Neck. Surg. 122 1996; 302-306.
16. Holinger LD. Etiology of stridor in the neonate, infant and child. Ann Otol Rhinol Laryngol 1980; 89:397-400. https://doi. org/10.1177/000348948008900502.

17. Weber PC, Kenna MA, Casselbrant ML. Laryngeal cysts: A cause of neonatal airway obstruction. Otolaryngol Head Neck Surg 1993; 109:129-34. https://doi.org/10.1177/01945 9989310900124.

18. Hsieh LC, Yang CC, Su CH, Lee KS, Chen BN, Wang LT. The outcomes of infantile vallecular cyst post CO2 laser treatment. Int J Pediatr Otorhinolaryngol 2013; 77:424-8. https://doi. org/10.1016/j.ijporl.2013.01.005.

19. Chen EY, Lim J, Boss EF, Inglis Jr AF, Ou H, Sie KC. Transoral approach for direct and complete excision of vallecular cysts in children. Int J Pediatr Otorhinolaryngol 2011; 75:1147-51. https://doi.org/10.1016/j.ijporl.2011.06.007. 